\title{
Systematic discovery of regulatory motifs in Fusarium graminearum by comparing four Fusarium genomes
}

\author{
Lokesh Kumar ${ }^{1,3}$, Andrew Breakspear ${ }^{2}$, Corby Kistler ${ }^{2 *}$, Li-Jun Ma $^{1 *}$, Xiaohui Xie ${ }^{3^{*}}$
}

\begin{abstract}
Background: Fusarium graminearum (Fg), a major fungal pathogen of cultivated cereals, is responsible for billions of dollars in agriculture losses. There is a growing interest in understanding the transcriptional regulation of this organism, especially the regulation of genes underlying its pathogenicity. The generation of whole genome sequence assemblies for $\mathrm{Fg}$ and three closely related Fusarium species provides a unique opportunity for such a study.

Results: Applying comparative genomics approaches, we developed a computational pipeline to systematically discover evolutionarily conserved regulatory motifs in the promoter, downstream and the intronic regions of $\mathrm{Fg}$ genes, based on the multiple alignments of sequenced Fusarium genomes. Using this method, we discovered 73 candidate regulatory motifs in the promoter regions. Nearly $30 \%$ of these motifs are highly enriched in promoter regions of $\mathrm{Fg}$ genes that are associated with a specific functional category. Through comparison to Saccharomyces cerevisiae (Sc) and Schizosaccharomyces pombe (Sp), we observed conservation of transcription factors (TFs), their binding sites and the target genes regulated by these TFs related to pathways known to respond to stress conditions or phosphate metabolism. In addition, this study revealed 69 and 39 conserved motifs in the downstream regions and the intronic regions, respectively, of $\mathrm{Fg}$ genes. The top intronic motif is the splice donor site. For the downstream regions, we noticed an intriguing absence of the mammalian and Sc poly-adenylation signals among the list of conserved motifs.

Conclusion: This study provides the first comprehensive list of candidate regulatory motifs in $\mathrm{Fg}$, and underscores the power of comparative genomics in revealing functional elements among related genomes. The conservation of regulatory pathways among the Fusarium genomes and the two yeast species reveals their functional significance, and provides new insights in their evolutionary importance among Ascomycete fungi.
\end{abstract}

\section{Background}

Collectively, fungal species within the genus Fusarium are among the most important group of plant pathogens, causing disease in nearly every agriculturally cultivated plant [1]. Mycotoxins produced by Fusarium species also pose a significant hazard to food safety and human health $[2,3]$. The economic and scientific importance of these fungi has led to whole genome sequencing and functional studies of multiple economically important and phylogenetically related Fusarium species

\footnotetext{
* Correspondence: hckist@umn.edu; lijun@broadinstitute.org; xhx@ics.uci.edu 'Broad Institute, Cambridge, MA, USA

USDA ARS Cereal Disease Laboratory, University of Minnesota, St Paul, MN,
} USA including, F. graminearum ( $F g), F$. verticillioides ( $F v)$, F. oxysporum (Fo), and F. solani [4-8]. Such rich genomic resources enable the discovery of many biological features related to the genetic mechanisms of organism adaptation and pathogenicity for these species [9-11].

As functional elements, transcription factor binding sites often evolve at a much slower rate than neutral sequences, and therefore they often stand out from the surrounding sequences by virtue of their greater levels of conservation. This property enables the recognition of these conserved elements through comparative genomics. Previous work has demonstrated the power of comparative genomics for discovering novel regulatory motifs in yeasts [12], Plasmodium [13], flies [14],

\section{Biomed Central}


mosquitoes [15] and mammals [16-18]. Even though there is growing body of knowledge about pathogenicity related gene regulation in the $F g$ genome, including spore germination [10] and adaptation to the host environment [11], very little is known about the transcription factors involved in the process and the regulatory elements targeted by these transcription factors. Comparative Fusarium genomics enables the generation of large-scale unambiguous alignments [8] among the four sequenced Fusarium genomes. The total divergence among these four genomes, measured by the total branch length of the tree connecting the four species is 0.35 (i.e. 0.35 substitutions per base, roughly the distance between human and dog). This distance is similar to that used successfully for identifying regulatory elements in other species $[12,18]$.

Here we describe a comparative genomics approach to systematically discover potential regulatory elements based on the sequence conservation of the non-coding regions among four sequenced Fusarium genomes (Figure 1). The results were supported by several lines of evidence, including: 1) co-regulation of subsets of genes that share the common regulatory elements in the promoter regions based on microarray expression data; 2) conservation of some of the potential transcription factor (TF) binding sites, the TFs themselves and the target genes regulated by these TFs compared to transcriptional regulatory networks described in the two model systems, Saccharomyces cerevisiae (Sc) and

$$
\begin{array}{|c|}
\hline \begin{array}{c}
\text { Generating multiple sequence alignments } \\
\text { among F. graminearum, F. verticillioides, } F . \\
\text { oxysporum, and F. solani }
\end{array} \\
\begin{array}{c}
\text { Calculating Motif Conservation } \\
\text { Score (MCS) of all 7-mers }
\end{array} \\
\hline \begin{array}{c}
\text { Optimizing significantly conserved } \\
\text { 7-mers by introducing degeneracy }
\end{array} \\
\hline \begin{array}{c}
\text { Clustering optimized motifs to } \\
\text { remove motif redundancy }
\end{array} \\
\hline \text { Testing enrichment of discovered } \\
\text { motifs in functionally related gene sets }
\end{array}
$$

Figure 1 Overview of the computational pipeline used for motif discovery.
Schizosaccharomyces pombe (Sp); and 3) identification of a splice donor site as the top intronic motif. As the first systematic survey of potential regulatory elements in Fusarium species, our study revealed that the most conserved regulatory elements from both upstream and intronic regions span distantly related fungal species, indicating that these elements are under strong functional constraint by negative selection and confirming that many discovered motifs likely have an essential biological function.

\section{Results}

\section{Motif discovery by comparing four Fusarium genomes Alignment}

The whole-genome alignments of the four Fusarium species were generated using PatternHunter (see Methods) and further divided into three functionally distinct genomic regions - promoters, downstream, and introns. The gene annotation of $F g$, the first sequenced [5] and betterannotated [6]Fusarium genome, was used as a reference. Because most of the $\mathrm{Fg}$ genes are computationally predicted and thus do not have well-defined transcriptional start or stop sites, we defined the promoter region to be up to 600 bp upstream of the translation start site (ATG) and not overlapping its neighboring gene. We chose 600 bp based on the mean intergenic distance of $\sim 1200 \mathrm{bp}$ in $\mathrm{Fg}$. Similarly, we defined the downstream region of a gene to be up to $600 \mathrm{bp}$ downstream of the stop codon without overlapping any other genes or potential promoter regions. Based on this definition, the downstream regions could be enriched for 3'UTR sequences, for which little is known in Fusarium genomes. To avoid redundancy, we further post-processed the promoter and downstream datasets to make sure that each genomic region is represented only once. Overall, the promoter data set contains 3.4 million aligned bases, whereas the intronic and downstream sets contain 1.3 and 2.3 million aligned bases respectively. Some segments could not be aligned in all four species, primarily because they represent new sequences recently inserted into the $F g$ genome, ancestral sequences deleted in one of the other species, or missing sequences in one of the draft genomes.

\section{Motif discovery}

A score metric called Motif Conservation Score (MCS) [18] was used to evaluate the conservation properties of all 7-mer motifs within each of these three datasets (see Methods and Additional file 1). We focused our initial efforts on 7-mers because most regulatory motifs are in the range of 6-10 bps, with 6-mers tending to give too many random matches, whereas 8 or higher-mers too few (Note also that 7-mers are only used for initial screening; the exact lengths of the motifs are actually determined later when we introduce degeneracy to motifs). For each dataset, we exhaustively searched for 
the presence of 7-mers and obtained two values for each of them (i) the total occurrences in the reference genome $F g(N)$, and (ii) the number of occurrences that are conserved among all four genomes in the aligned regions $(k)$. We called a motif occurrence conserved if the identical heptamer in the aligned regions occurs within a window of $-5 \mathrm{bp}$ to $+5 \mathrm{bp}$ compared to the reference position. The $5 \mathrm{bp}$ extension at each side was introduced to account for potential errors in local sequence alignments. We next evaluated the MCS value for each heptamer, which essentially represents the difference in conservation of a heptamer from the expected conservation of a random heptamer (see Methods). For instance, the 7-mer CACGTGA occurred $N=264$ times in the $\mathrm{Fg}$ genome of the promoter dataset. Among the 264 sites in $F g, \mathrm{k}=151$ are also conserved in the corresponding orthologous regions of the three other species, corresponding to a conservation rate (C.R.) of $57 \%$. By contrast the conservation rate for a random 7 -mer in the promoter dataset is only $p_{0}=3 \%$, which indicates that the CACGTGA motif shows a 19 fold enrichment in conservation rate, corresponding to an $\mathrm{MCS}=(k-$ $\left.N p_{0}\right) / \operatorname{sqrt}\left(N p_{0}\left(1-p_{0}\right)\right)=51$ s.d.

The distribution of the MCS values in each region is biased towards positive values (Figure 2 and Additional file 1, Figure S1), indicating that many heptamers are more conserved than would be expected from a completely random case. The lowest MCS value of a heptamer is $-4.4,-4.1$ and -2.4 in the promoters, downstream regions and introns respectively. We selected the absolute value of the lowest score in the promoters as the threshold to select over-conserved and thus potential regulatory motifs in all regions. If the sequences in the alignment evolved neutrally, the MCS scores would be symmetric and centered at zero. Thus, we would expect to see no heptamers with MCS values higher than the selected threshold for each region. In contrast, the selection step yielded 688 heptamers in the promoter region, 326 heptamers in the downstream region and 234 heptamers in the intronic region, corresponding to $4 \%, 2 \%$ and $1.4 \%$ of all heptamers in three regions respectively. We noticed that the heptamers identified from each region are largely non-overlapping with only 50 heptamers shared by all three regions (Additional file 1, Figure S2), suggesting that the identified motifs are mostly region-specific. The 50 shared heptamers likely result from partial overlap of functional regions due to incorrect annotation of a subset of genes, true signals shared by all three regions, or simply random matches.

\section{Adding Degeneracy}

Regulatory motifs typically contain certain variations without greatly affecting the overall binding affinity, as many of the selected heptamers are slight variations of each other. Therefore, we sought to identify the true motifs associated with the discovered heptamers by introducing degeneracy into certain positions of the heptamers. If the motif, after introducing the degeneracy, is a closer representation of a regulatory element, the MCS score of the modified motif should be higher. Therefore, we developed a greedy algorithm to systematically search for the pattern of degeneracy that can lead to an increase in the MCS score of the modified motifs (see methods and Additional file 1). Briefly, the program randomly selected a base position in the heptamer and added a degenerate character that is consistent with the nucleotide at that position. If this change increased the MCS in the updated motif, we repeated this process for one more base position. As this greedy approach is susceptible to local maxima, we repeated it ten times and selected the degenerate motif with the highest MCS from all the trials. We used this approach on the heptamers in the decreasing order of their MCS values and ignored a heptamer if it could be specified by one of the degenerate motif derived from earlier heptamers. This step finally gave 326 motifs in the promoter regions, 206 in the downstream and 114 in the intronic regions. Some of the heptamers were able to increase their score by as much as $300 \%$. ACGCGTC in promoters, for example, increased its MCS from an original value of 14.3 to 40.6 as it degenerated into nCGCGnC ('n' represents any nucleotide). The complete list of degenerate motifs is available from our project website http://www. broadinstitute.org/annotation/genome/fusarium_group/ SupplementalMaterials.html.

The MCS score measures the conservation across all sites of a motif; therefore it should not be very sensitive to the specific definition of promoters we used as long as the distribution of the motif in the promoters is not biased toward long distances. To illustrate this, we generated two additional promoter datasets using the distance of $400 \mathrm{bp}$ and $1000 \mathrm{bp}$ respectively for the motif discovery. For the 400 bp dataset, we discovered 288 motifs (prior to clustering), of which 286 are present in the 326 motifs discovered in the $600 \mathrm{bp}$ dataset. For the 1000 bp dataset, we discovered 394 motifs, which include all of the 326 motifs discovered in the $600 \mathrm{bp}$ dataset. For these 394 motifs, more than $80 \%$ of all the sites are located within $600 \mathrm{bp}$ from gene starts with the highest density located at 70 bp from gene starts (Additional file 1, Figure S3). This suggests that the $600 \mathrm{bp}$ is a reasonable choice for the definition of promoter regions, although we note that distant motifs preferentially located far away from gene start sites are likely to be missed with this definition.

\section{Clustering}

The degenerate motifs, thus derived, were still redundant. Our next approach to more accurately predict potential regulatory elements was to further cluster 


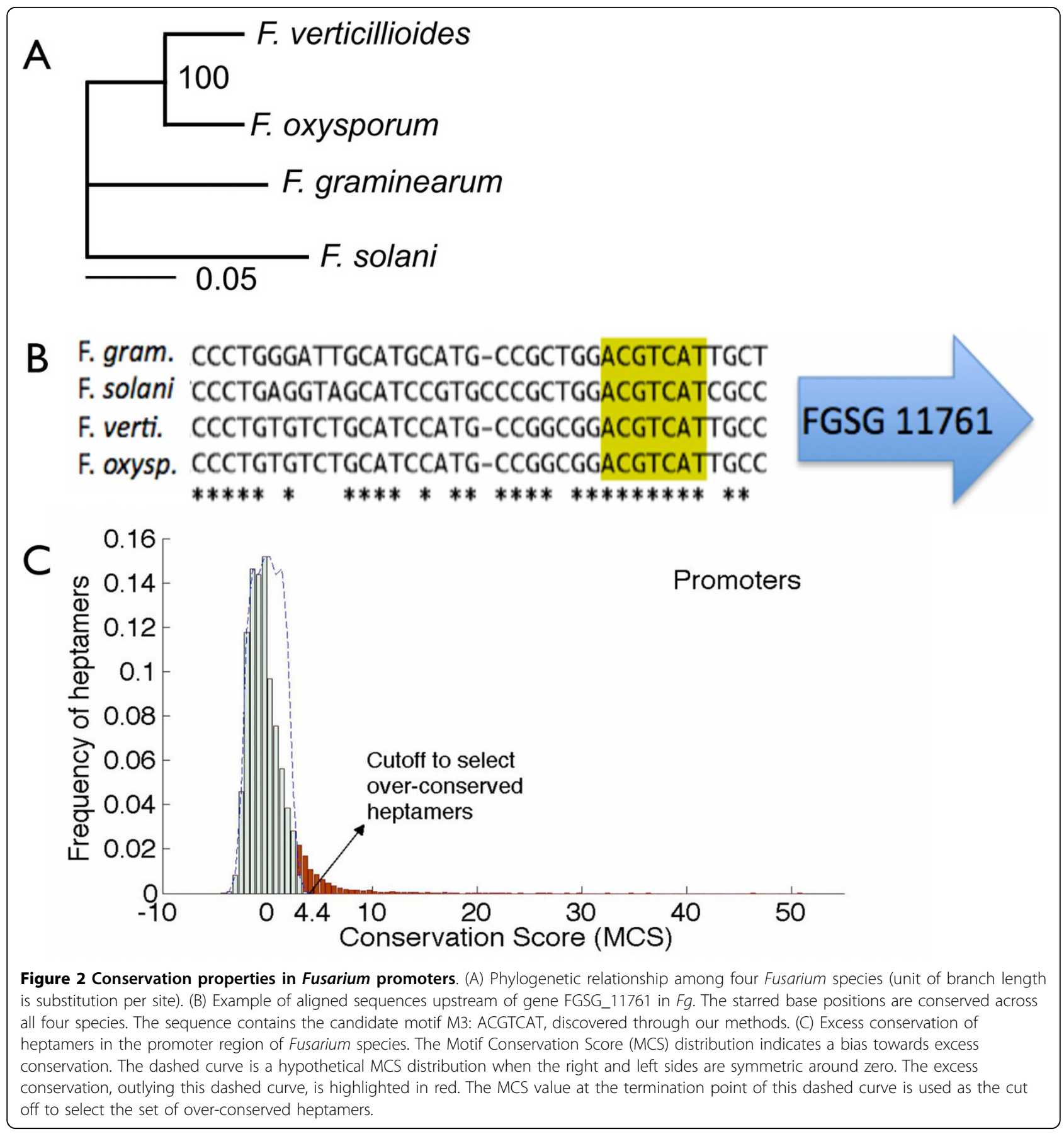

degenerate motifs using the Pearson correlation as a similarity measure for each pair of motifs (see Additional file 1). The Pearson correlation computes the sequence similarity between the equivalent position weight matrices [19] and quantifies it with a score from -1 to +1 ; with +1 for identical motifs. We grouped motifs in the same cluster if they were at least 0.75 correlated with the highest MCS motif in the cluster. From this final stage of our computational pipeline, we identified 73 clusters in promoters, 69 in the downstream and 39 clusters in the intronic region. Each cluster is represented by the highest scoring motif within that cluster. The list of candidate motifs from each cluster is shown in Table 1 and Additional file 1, Table S1.

\section{Association with functional gene clusters}

Since very little is known about the constituents of regulatory pathways in Fusarium species, we searched for 
Table 1 Top 30 discovered motifs in the promoter regions as ranked by MCS scores

\begin{tabular}{|c|c|c|c|c|c|c|c|c|c|}
\hline \multirow[t]{2}{*}{ ID } & \multirow[t]{2}{*}{ Top Motifs } & \multicolumn{4}{|c|}{$\begin{array}{l}\text { Genome wide statistics for the aligned } \\
\text { region }\end{array}$} & \multicolumn{4}{|c|}{ Functional category enrichment* } \\
\hline & & $N$ & & C.R. & MCS & $\begin{array}{l}\text { Most enriched } \\
\text { functional category }\end{array}$ & $\begin{array}{l}\text { Genes in functional } \\
\text { category }\end{array}$ & $\begin{array}{l}\text { Motif genes in } \\
\text { Fun-Group }\end{array}$ & $\begin{array}{l}\text { Enrichment } \\
\text { Score }\end{array}$ \\
\hline$\overline{\mathrm{M} 1}$ & CCCCnC & 4734 & 1462 & 0.30 & 93.5 & Nucleolus 1 & 128 & 75 & 11.9 \\
\hline M2 & CACGTG & 623 & 315 & 0.50 & 66.4 & Ribosome 2 & 88 & 47 & 24.3 \\
\hline M3 & CGTCAY & 1970 & 468 & 0.23 & 41.6 & Ribosome 2 & 88 & 53 & 5.0 \\
\hline M4 & CGCGnC & 2195 & 461 & 0.21 & 40.6 & Nucleus 2 & 341 & 173 & 9.6 \\
\hline M5 & CGCCnC & 3007 & 525 & 0.17 & 37.2 & - & - & - & - \\
\hline M6 & CCCCnG & 2985 & 509 & 0.17 & 36.0 & - & - & - & - \\
\hline M7 & CCnCCA & 5444 & 767 & 0.14 & 35.4 & Nucleus 2 & 341 & 178 & 7.9 \\
\hline M8 & CTCCnC & 4829 & 673 & 0.13 & 35.2 & Peroxisome 2 & 32 & 24 & 5.4 \\
\hline M9 & CnCCGMC & 1857 & 357 & 0.19 & 33.8 & Nucleolus 1 & 128 & 43 & 12.2 \\
\hline M10 & $\mathrm{CnCnCCC}$ & 4201 & 565 & 0.13 & 31.8 & Nucleus 3 & 159 & 110 & 6.2 \\
\hline M11 & CCAATnA & 1105 & 212 & 0.19 & 30.6 & - & - & - & - \\
\hline M12 & GCnnCGC & 2624 & 385 & 0.14 & 29.3 & Cytoskeleton 1 & 22 & 15 & 5.3 \\
\hline M13 & ACGYSAC & 797 & 173 & 0.21 & 27.9 & Nucleus 2 & 341 & 150 & 6.7 \\
\hline M14 & CCSGCC & 1520 & 258 & 0.16 & 26.5 & - & - & - & - \\
\hline M15 & CnGCnCC & 3170 & 421 & 0.13 & 26.0 & - & - & - & - \\
\hline M16 & CnTCnCC & 5870 & 636 & 0.10 & 26.0 & - & - & - & - \\
\hline M17 & ACCCCG & 761 & 152 & 0.19 & 25.9 & - & - & - & - \\
\hline M18 & CGGnCCG & 369 & 93 & 0.25 & 25.2 & - & - & - & - \\
\hline M19 & AAAAA & 6137 & 668 & 0.10 & 24.5 & Nucleolus 1 & 128 & 113 & 19.0 \\
\hline M20 & CCnCnTC & 5505 & 545 & 0.09 & 24.4 & - & - & - & - \\
\hline M21 & AAAWTTY & 853 & 169 & 0.19 & 23.7 & Nucleolus 1 & 128 & 101 & 31.4 \\
\hline M22 & TGCCCC & 777 & 139 & 0.17 & 22.9 & Nucleus 2 & 341 & 98 & 5.4 \\
\hline M23 & GnGGCT & 2449 & 321 & 0.13 & 21.5 & - & - & - & - \\
\hline M24 & GCGCnC & 1673 & 239 & 0.14 & 21.2 & Nucleus 2 & 341 & 196 & 6.4 \\
\hline M25 & TCCCnC & 4141 & 432 & 0.10 & 20.8 & - & - & - & - \\
\hline M26 & YGATAAG & 393 & 85 & 0.21 & 20.2 & Nucleolus 1 & 128 & 38 & 10.9 \\
\hline M27 & CGACnnC & 3725 & 399 & 0.10 & 19.9 & - & - & - & - \\
\hline M28 & CCGCnGn & 1874 & 245 & 0.13 & 19.7 & - & - & - & - \\
\hline M29 & CCTCGGY & 381 & 80 & 0.20 & 18.9 & Peroxisome 2 & 32 & 22 & 13.5 \\
\hline M30 & AnnCCAC & 3708 & 373 & 0.10 & 18.6 & - & - & - & - \\
\hline
\end{tabular}

Each motif shown here is the representative motif (i.e. the one with highest MCS score) from each cluster. $N$ and $k$ respectively represent the number of total occurrences in F.g. and the number of occurrences conserved in all four Fusarium genomes. C.R. is the conservation rate of the motif (= k/N) and MCS is the motif conservation score. * The promoter enrichment statistics is for the motif with the highest enrichment score (10 ${ }^{\text {th }}$ column) within each cluster shown in the $1^{\text {st }}$ column. The names of the functional categories are derived from the GO annotation of $F . g$. The number associated with each category represents the cluster as identified by the k-means clustering on the expression data within each category. Enrichment score is in the unit of -log ${ }_{10}(\mathrm{p}$-value).

potential associations of promoter motifs with specific gene functions using a combination of Gene Ontology (GO) annotation [20] and expression profiles [10]. For each motif, we searched for the set of genes that contain the motif or its reverse pair in its promoter region. Each gene set associated with particular promoter motif is divided into GO category group per their cellular functions. Genes within each GO category group were further sub-grouped into clusters of genes with similar expression profiles using $\mathrm{K}$-means clustering of previously published expression data for conidial germination [10]. Gene expression was measured in fresh conidia $(0 \mathrm{~h})$ and at three other developmental milestones: spore swelling $(2 \mathrm{~h})$, germ tube emergence and elongation $(8 \mathrm{~h})$ and hyphal branching $(24 \mathrm{~h})$.

The GO cellular annotation provides a total 21 functional categories over $13332 \mathrm{Fg}$ genes. Each category was further divided into 2 to 5 clusters within each GO category through $\mathrm{K}$-means analysis and resulted in a total of 68 sub-clusters (Called FunGroup). A functional survey for each cluster was carried out using MIPS FunCat $(5,6)$. Gene lists, expression profiles and functional analysis for each cluster can be found on our project website shown above.

Even though, only limited expression data was used for this analysis, the resulting clusters reflect the 
functional association expected during spore germination. For example, cytoskeleton cluster 1 is enriched for genes involved in budding, cell polarity and filament formation (MIPS category 43.01.03.05; $P=5.85 \mathrm{E}-21$ ), such as actin and microtubule cytoskeleton genes necessary for polarized growth in filamentous fungi [21]. As expected, genes belonging to this cluster are up-regulated between $2 \mathrm{~h}$ and $8 \mathrm{~h}$, following the switch to polar growth and germ tube emergence. Even though the FunGroups resulting from this analysis only capture part of the transcriptome, their significant association confirmed the potential functionality of the promoter motifs discovered by our computational pipeline. The significance of enrichment for motif containing genes within each FunGroup was quantified using the hypergeometric distribution (see methods). Even though the promoter motifs are clustered based on sequence similarity, we tested each motif individually within all the 68 genes subgroups to increase specificity, knowing that a single base difference may produce slightly different target gene set. At $\mathrm{P}$-value $<10^{-3}, 40$ motif clusters had at least one motif that is significantly enriched. At P-value $<10^{-5}, 22$ motif clusters (30\%) showed strong enrichment (Figure 3). Notable examples include the palindrome motif M2 (CACGTG), strongly enriched in the second expression cluster of the Cellular Ribosome GO category, and the M29 motif (CCTCGGY), highly enriched in the second expression cluster of the Cellular Peroxisome category. Some motifs, like M1, M19 and M21, are enriched in multiple functional categories, suggesting their essential roles in gene regulation.

We further tested the significance of the enrichment between the promoter motifs and FunGroups through a control experiment, in which we generated a set of control motifs by randomly permuting the bases within each discovered promoter motif. The enrichment analysis on the control motifs resulted in only two enriched clusters $(2 \%)\left(\mathrm{P}\right.$-value $\left.<10^{-5}\right)$, much less than 22 as for the discovered motifs (30\%). This test may suggest that the false discovery rate is below $9 \%$, ( $\mathrm{P}$ value $<10^{-5}$ ), and the enrichment of the motif containing genes within FunGroups are likely due to biologically bona fide associations.

\section{Association with known transcriptional pathways}

Furthermore, we are interested in understanding specific functions and potential regulatory pathways associated with the identified promoter motifs in $\mathrm{Fg}$ by comparing the motif and motif containing genes to known TF binding sites and their target genes. We have focused such comparison with two yeast systems $S c$ and $S p$, and extended this to other known ascomycete fungal TFs. Based on the motif comparison [22] (see Additional file $1)$, we found $16(21 \%)$ discovered motifs at E-value $<10^{-7}$

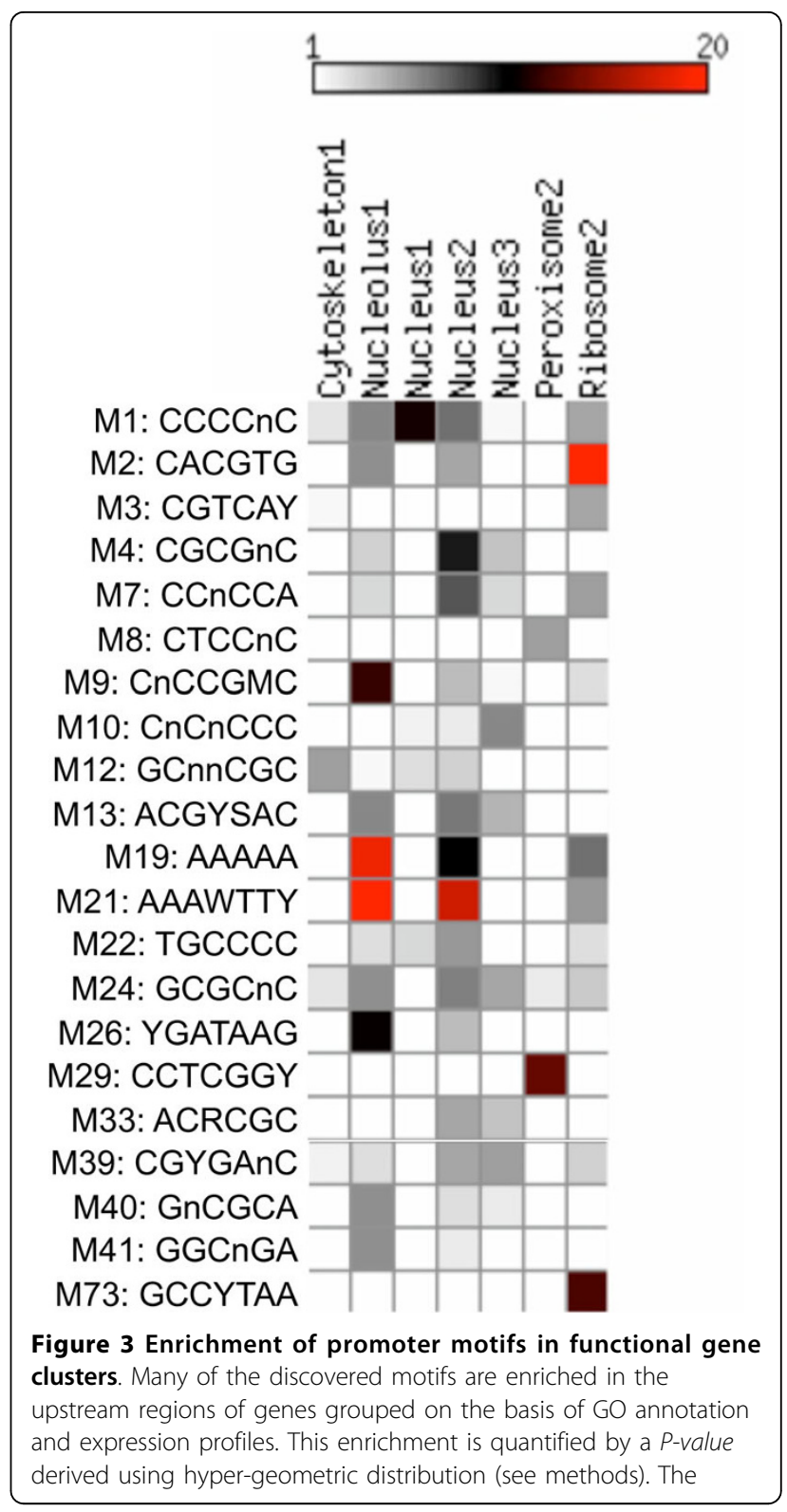

(Figure 4) or about half of the motifs at, E-value $<10^{-5}$ share sequence similarity to the binding sites reported in $S$. cerevisiae $(S c)$ [23]. If the sequence conservation truly reflects the functional association of these motifs, we were interested in determining whether these motifs are regulated by orthologous TFs and whether the target genes also are conserved in these distantly related fungal species.

\section{Phosphate metabolism}

The most significant match between $F g$ motifs and the Sc TF binding site is the motif M2 (CACGTG), which has the second highest MSC score in our list and matches the Pho4 motif in Sc. Pho4 is a basic helixloop-helix (bHLH) TF that functions during phosphate 


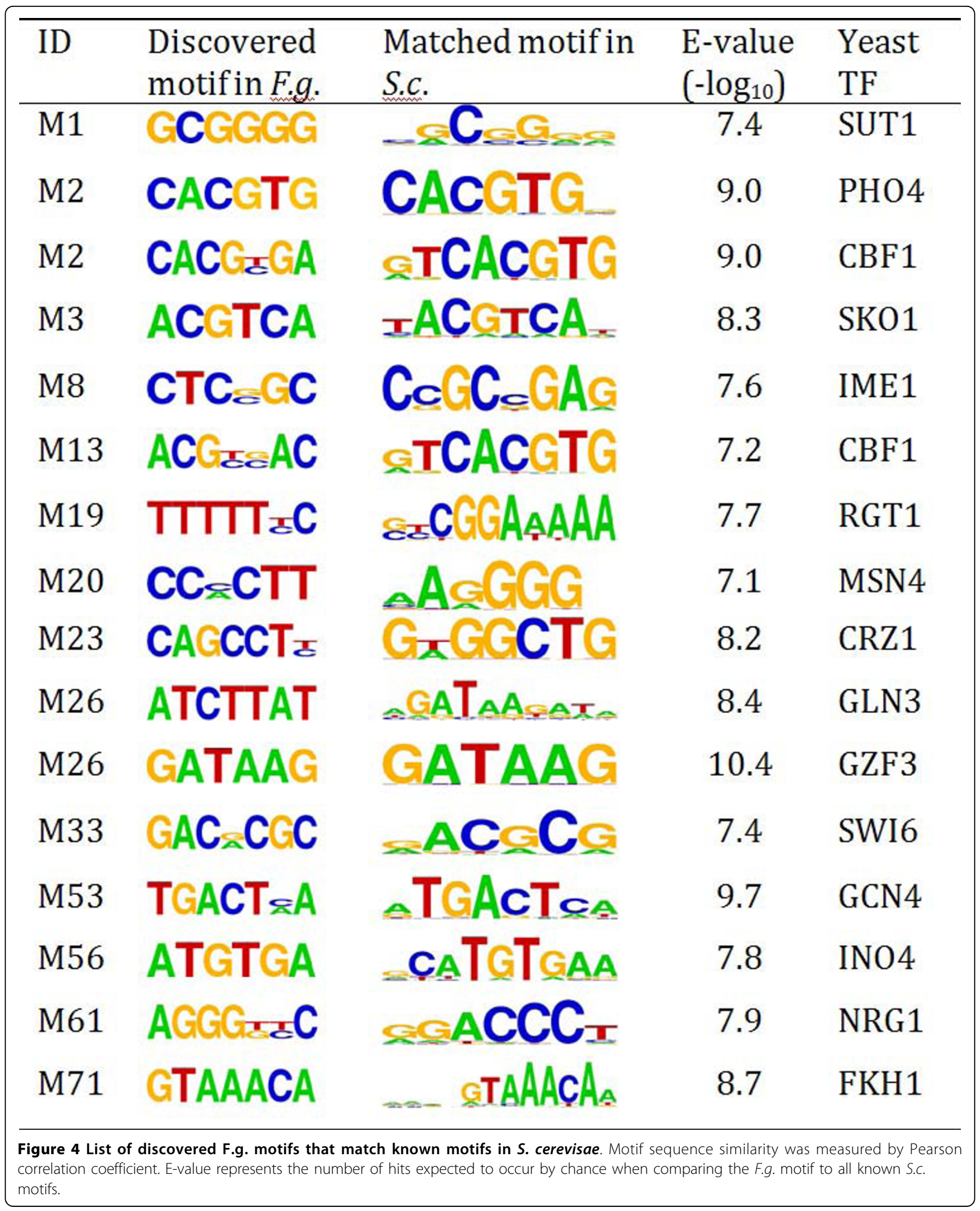


(Pi) starvation [24]. In high phosphate growth conditions Pho4 is phosphorylated and present only in the cytoplasm. Under phosphate starvation, dephosphorylated Pho4 enters the nucleus and activates pho genes involved in response to phosphate starvation [25]. There is a Pho4 ortholog in each Fusarium genome (FGSG_00545, FOXG_00510, JGI_32105, and FVEG_01003). More than 60\% of the Pho4 interacting genes in $S c$ [26] have a homologous gene in $F g$ (BLAST $1 \mathrm{e}-5$ ) and the potential binding site M2 is significantly enriched among this set of genes (Figure 5). As demonstrated for both Neurospora crassa (Nuc1) [27] and Aspergillus nidulans (PalcA) [28], our study confirms that the Pho4 orthologs regulate the PHO system in filamentous fungi through the same TF and TF binding site, strongly suggesting the biological importance of this particular pathway.

Interestingly, through the GO category enrichment test, motif M2 (CACGTG) is highly enriched in the upstream promoter regions of genes in ribosome cluster 2 (Figure 3). Within this cluster, 44 out of 47 genes are predicted ribosomal proteins. Remarkably, we observed the expression profile for genes contained in ribosome cluster 2 peaking sharply at 2 and $8 \mathrm{~h}$, periods of spore activation and germ tube emergence where a high level of ribosome biogenesis would be required to cope with an increased demand for nascent proteins. Even though, the components of the ribosome have not been identified as targets of Pho4 in yeast [24], more than $30 \%$ of the 155 proteins interacting with Pho4 TF [26] are classified as ribosome function related according to SlimGO annotation. In $N$. crassa, 7 ribosomal proteins are regulated by $N u c-1$, and 4 of them contain CACGTG in their promoter regions [29]. The CACGTG motif is also enriched in the upstream promoter regions of genes in cytoskeleton cluster 1,4 and cortex cluster 3 . Therefore we hypothesize that in addition to Pi metabolism, Pho4 TF also regulates genes belonging to diverse functional categories including protein biosynthesis and the cell cycle.

\section{Stress response}

The other top scoring motif M3, ACGTCA, matches the binding site of $S k o 1$ in $S c$ and Atf1 in $S p$, both of which are known TFs that regulate response to environmental

\begin{tabular}{|c|c|c|c|c|c|c|c|c|c|c|}
\hline 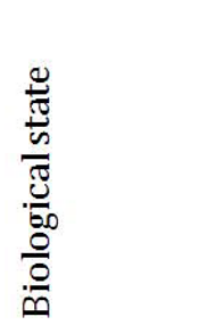 & 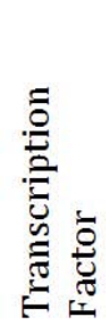 & 范 & 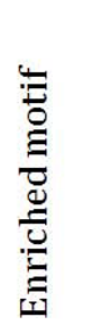 & 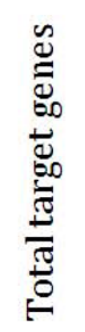 & 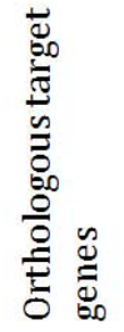 & 吾 & 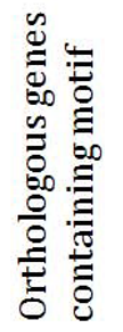 & 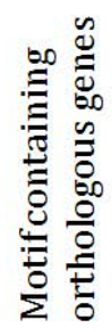 & 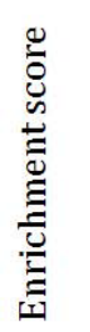 & 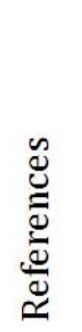 \\
\hline $\begin{array}{l}\text { S. cerevisiae } \\
\text { Phosphate } \\
\text { metabolism }\end{array}$ & $\begin{array}{l}\mathrm{PHO} \\
4\end{array}$ & CACGTG & M2: & 154 & 93 & 1290 & 449 & 29 & 3.1 & [24] \\
\hline $\begin{array}{l}\text { S. cerevisiae } \\
\text { ESR induced }\end{array}$ & $\begin{array}{l}\text { MSN } \\
2 / 4\end{array}$ & AAGGGG & $\begin{array}{l}\text { M22 } \\
\text { 플 }\end{array}$ & 139 & 85 & 5263 & 1222 & 57 & 3.7 & $\begin{array}{l}{[27,} \\
34]\end{array}$ \\
\hline $\begin{array}{l}\text { S. pombe } \\
\text { CESR } \\
\text { induced }\end{array}$ & Atf1 & TACGTCA & M3*: & 314 & 93 & 3713 & 750 & 42 & 3.8 & [32] \\
\hline $\begin{array}{l}\text { S. cerevisiae } \\
\text { Osmotic } \\
\text { response }\end{array}$ & $\begin{array}{l}\text { SKO } \\
1\end{array}$ & TACGTCA & M3*: & 18 & 9 & 4297 & 945 & 8 & 2.7 & [40] \\
\hline $\begin{array}{l}\text { S. cerevisiae } \\
\text { ESR } \\
\text { repressed }\end{array}$ & - & $\begin{array}{l}\text { AAAAWT-- } \\
\text { TTT }\end{array}$ & $\begin{array}{l}M 21 \\
A A\end{array}$ & 590 & 359 & 3809 & 963 & 225 & 24.9 & $\begin{array}{l}{[31,} \\
40]\end{array}$ \\
\hline $\begin{array}{l}\text { S. pombe } \\
\text { CESR }\end{array}$ & - & - & M19* & 459 & 154 & 6709 & 1532 & 132 & 15.3 & $\begin{array}{l}{[32,} \\
40]\end{array}$ \\
\hline
\end{tabular}


stress [30,31]. Even though both Sko1 and Atf1 are known to use the same TF binding sites (ACGTCA), their roles in stress defense and the set of genes they regulate have significantly diverged. Skolp in Sc regulates a small set of genes in response to osmotic shock [30], while its ortholog Atf1 in fission yeast $S p$ regulates a larger gene set induced by environmental stress under diverse conditions that define Atf1 as a core environmental stress response (CESR) regulator [32]. There is a Sko1/Atf1 ortholog in each Fusarium genome (FGSG_10142, FVEG_02866, FOXG_05265 and JGI_69482), although the Fusarium genes are more similar to Atf1 (1e-32 between FGSG_10142 and Atf1, 4.5e-9 between FGSG_10142 and Sko1). About 20 Sko1 target genes were identified in Sc [30], 9 of which are conserved in $\mathrm{Fg}$. The binding site, M3, is conserved in 8 of them. Similar conservation and motif enrichment result is also observed among the $314 S p$ induced CESR gene set (Figure 5). It is likely that the Sko1/Atf1 homo$\log$ in Fg regulates both CESR as in Sp and the osmotic defense response as reported in Sc. Consistent with this hypothesis, Sp is reported to be sensitive to osmotic stress, while both Fg and $\mathrm{Sc}$ are resistant to osmotic stress [33], which may indicate that the osmotic regulation through the Sko1 pathway may have either evolved after the split of the Sp lineage, or lost in Sp. The smaller genome size and number of genes in the two yeast systems implies that the yeasts are streamlined derivatives of a common ascomycete ancestor.

In $S c$, the CESR is regulated through a different pathway using the duplicated TF gene pair MSN2/4 which bind to a sequence that differs from the Sko1/Atf1 binding site. A single $M S N$ homologous gene is present in each Fusarium genome (FGSG_06871, FVEG_05115, FOXG_01955, and JGI_102564). The Fg genes homologous to the Sc CESR pathway genes are also enriched, but for a slightly different motif than the MSN2/4 binding sites (Figure 5). Based on all these observations, we infer that, in response to environmental stresses, the filamentous fungus $\mathrm{Fg}$ employs the combined regulatory pathways used in both yeast systems.

Large sets of genes are also repressed under environmental stress in $S c$ and $S p$ respectively [31,32] and the same TF binding site was reported in both systems. About $60 \%$ and $33 \%$ of the repressed genes, from $S c$ and $S p$ respectively, share homologous genes in $F g$. Two very similar motifs M19 and M21 are significantly enriched in two homologous sets accordingly (Figure 5). Clearly similar regulatory mechanisms are operating among these diverse fungal genomes, while there are more genes involved the regulation in the filamentous fungus $\mathrm{Fg}$ comparing to the rather streamlined yeast regulatory systems. The functional association study also revealed that these two motifs (M19 and M21) are enriched in the Cellular Ribosome GO category, cluster 2 (Figure 3 ). In rapidly growing yeast cells, about $60 \%$ of total transcription is devoted to ribosomal RNA, and $50 \%$ of RNA polymerase II transcription is devoted to ribosomal proteins (RPs) [34]. Therefore repression of the genes encoding ribosomal proteins may ensure the economic use of resources under stressed conditions.

As one of the initial efforts to study whole genome regulation in filamentous fungi, it is perhaps not surprising to see many of the motifs discovered from our study have no matches in S. cerevisiae, including the top-scoring motif M1 (GCGGG). However, comparing these motifs to known TF binding sites from other filamentous fungi identified the M1 motif as highly similar to the binding site of transcription factor CreA (G/ CPyGGGG), a well known repressor responsible for carbon catabolite repression in Aspergillus nidulans [35]. By searching for other known Aspergillus transcription factor binding sites within the list of discovered Fusarium promoter motifs, we found two additional matches, including motifs M5 (GCCARG) and M26 (YGATAAG), which show high similarity to the $P a c C$ and the AreA DNA-binding sites respectively. PacC plays an important role in mediating the response to ambient $\mathrm{pH}$, while AreA belongs to the GATA family of DNA-binding proteins that binds to HGATAR and mediates nitrogen metabolism repression [36].

\section{Discovered motifs in downstream and intronic regions}

In addition to the promoter motifs, our analysis also yielded a number of motifs showing significant conservation in the downstream and intronic regions (Additional file 1, Table S1), although the overall number is smaller and the motifs are less conserved when compared to the promoter region (Additional file 1, Figure S4). The most conserved intronic motif (GTNAGT, Additional file 1, Table S1), corresponding to a splice donor motif, is both abundant (6648 instances) and well conserved with C.R. (conservation rate) equal to $37 \%$. However, among the downstream conserved motifs we didn't find the mammalian polyadenylation signal (AATAAA). The polyadenylation hexamer, though abundant, is rarely conserved in the Fusarium genome. Among the downstream regions, we found a total of 1138 occurrences, only 11 of which are conserved (C.R.: 0.9\% and MCS = -3.69), which is even lower than the rate for a random hexamer (C.R.: 2.7\%). The known polyadenylation signals in $S c$, TATATA and TTAAGAAC $[37,38]$, also have a low MCS value of -1.8 ( 6 conserved from a total of 409 instances) and -0.3 ( 0 conserved from a total of 9 instances) respectively, suggesting that a different polyadenylation signal may be used in the Fusarium genomes. 
The discovered downstream motifs and the splice signals in the introns show a strong directional bias, while the promoter motifs have similar conservation rate in both the forward and reverse strands (Additional file 1, Figure S5). This directional specificity is consistent with the function of the downstream motifs and the splice signals in post-transcriptional processes, in contrast with the promoter motifs that act at the level of DNA, as binding sites during transcription.

\section{Discussion}

There is limited knowledge about transcriptional regulation among filamentous fungi. This study is the first attempt to systematically characterize sequence signatures that are conserved through evolution and may have the potential to function as regulatory elements among several Fusarium species, a group of agricultural important filamentous fungi. The study results in a total of 73 conserved elements in promoter regions. These promoter motifs are enriched in the upstream region of the genes involved in specific cellular functions, indicating their potential functional association with specific biological processes and validating the biological significance of our discovery. Interestingly, we observed the conservation among Fusarium species and two yeast model systems, $S c$ and $S p$ for some TFs, their binding sites and target genes regulated by these TFs for some important pathways, including phosphate metabolism and stress response. Both computational and experimental approaches were used to define modularity of regulatory network [39]. The conservation of potential regulatory elements accompanying certain conserved modules was also reported $[39,40]$.

Constantly exposed to a wide range of environmental changes, including hostile conditions, environmental stress response is essential for the survival of fungi. In that sense, it is not surprising to observe a significant conservation among both induced and repressed gene sets in response to environmental stresses across divergent lineages. Our study suggests that the core environmental stress response (CESR) is a highly conserved physiological mechanism that protects cells and organisms from stressful changes in their environment. The conservation between $S c$ osmotic stress response pathway and the $F g$ gene sets suggests that this regulatory pathway may have evolved after the split of Schizosaccharomyces, or was lost in this Ascomycete basal lineage. The more complete list of genes that are repressed under ESR in $\mathrm{Fg}$ also suggests that both yeast systems have independently reduced portions of the pathway through evolution.

The focus of this study is on short regulatory motifs with size around $7 \mathrm{bp}$. Therefore translation-related motifs, which are typically longer, are likely missed by our method. In addition, the translation-related motifs such as internal ribosome entry site (IRES) and upstream ORFs (uORFs) typically observe different conservation characteristics than the transcription-related motifs, since what is preserved for IRES is its secondary structure and what is preserved for uORFs is their short ORF structure. Detecting these translation-related motifs likely will require a new conservation measure that can take these specific characteristics into account. It is interesting to note that a motif containing ATG (ATGACGN with the MCS >30), which could encode the start codon of uORFs, is detected in the upstream regions.

\section{Conclusions}

We developed a computational pipeline to systematically discover regulatory motifs in $F g$ by comparing its genome to three other closely related Fusarium genomes. Our analysis yielded 73 candidate motifs in promoter regions, 69 in the downstream regions, and 39 in the intronic regions. Out of the 73 motifs discovered in the promoter regions, 22 showed strong enrichment in functionally related gene clusters, and 16 showed strong sequence similarity to known motifs in $S c$, altogether corresponding to $41 \%$ of the discovered motifs, suggesting that many of the motifs are likely true functional elements. Comparison of these motifs with known yeast TF binding sites revealed a significant conservation for signals involved in phosphate metabolism and ESR pathways, providing the first look into the regulation of these biological processes in $\mathrm{Fg}$. Such conservation across millions of years of evolution since the divergence of yeast and $F g$ indicates the functional importance of these regulatory pathways. The analysis presented here demonstrates the power of comparative genomics for discovering functional elements in the Fusarium genome. As more genomes and better annotation of genes become available, the list of regulatory motifs presented here can be further refined.

\section{Methods}

\section{Genome alignments and annotation}

Local-alignment anchors were detected using PatternHunter (1e-10) [41]. Contiguous sets of anchors with conserved order and orientation were chained together within a $10 \mathrm{~kb}$ distance. The multiple alignments for all the syntenic regions that cover a common segment on the reference genome $F g$ were conducted using Mlogan [42] We used the annotation of $F g[5,6]$ as a reference to define the coding sequence versus intergenic (600 bp upstream of the start codon and 600 bases downstream of the stop codon) and intronic regions in the aligned regions. For the intronic regions, we extended sequences by $2 \mathrm{bp}$ both upstream and downstream in order to include the intron splice sites. 


\section{Motif Conservation Score}

The motif conservation score (MCS) for the $\mathrm{i}^{\text {th }}$ heptamer $\mathrm{H}_{i}$ is computed using the following function:

$$
M_{C}=\frac{K_{i}-N_{i} P_{O}}{\sqrt{N_{i} P_{O}\left(1-P_{O}\right)}}
$$

where $K_{i}$ is the number of conserved occurrences and $N_{i}$ is the number of total occurrences of $\mathrm{H}_{\mathrm{i}}$ in the aligned genome of $F g$ with $F o, F v$ and Fs. $P_{o}$ is the fraction of total conserved occurrences among the total occurrences of all heptamers. The same function is used to compute the MCS for degenerate motifs. However, the $P_{o}$ is calculated differently. Since $P_{o}$ denotes the average conservation rate of a motif $\mathbf{m}$, we scan 5000 random heptamers in the genome and calculate its conservation as per the degeneracy profile of $\mathbf{m}$. This conservation score is then used to compute the average conservation rate for a motif whose degeneracy profile is the same as $\mathbf{m}$ (See Additional file 1 for details).

\section{Degeneracy}

We used consensus sequences to represent regulatory motifs. The sequences are spread over 11 alphabets, consisting of four nucleotides A, C, G, T, the six twofold degenerate characters $S=[C$ or $G], W=[A$ or $T]$, $\mathrm{Y}=[\mathrm{C}$ or $\mathrm{T}], \mathrm{R}=[\mathrm{A}$ or $\mathrm{G}], \mathrm{M}=[\mathrm{A}$ or $\mathrm{C}], \mathrm{K}=[\mathrm{G}$ or $\mathrm{T}]$, and the four-fold degenerate character $\mathrm{N}=[\mathrm{A}, \mathrm{C}, \mathrm{G}$, or $\mathrm{T}]$. A motif $\mathbf{m}$ occurs in the $F g$ genome when each nucleotide in the genome satisfies the corresponding degenerate character in the consensus sequence of $\mathbf{m}$. We used a greedy approach to discover motifs with higher MCS values by adding degeneracy as described in the main text and Additional file 1.

\section{Clustering}

We clustered the degenerate motifs as per their sequence similarity. We quantify the similarity between two motifs by the Pearson correlation of their equivalent position weight matrices. The weight matrices represent the frequencies of different nucleotides at each base. For example, if the base is $\mathrm{W}=(\mathrm{A}$ or $\mathrm{T})$, its corresponding column in the matrix will be represented as $[0.5,0,0$, 0.5 ] in the order of A, C, G and T. We computed the Pearson correlation coefficient between two positional weight matrices as described in Xie et al [18].

\section{Enrichment}

The enrichment, or overlap, between two sets of genes is computed by assuming an underlying hyper-geometric model. Given the total number of $\boldsymbol{N}$ genes in $F$, with $\boldsymbol{F}$ number of genes in a functional category set, $M$ number of genes that contains a motif $\mathbf{m}$, and $\boldsymbol{K}$ number of genes that are shared by $\boldsymbol{F}$ and $\boldsymbol{M}$, the P-value of enrichment of motif $\mathbf{m}$ in the set $\boldsymbol{F}$ is computed as:

$$
P-\text { value }=\sum_{i=K}^{\min (F, M)} \frac{\left(\begin{array}{l}
F \\
i
\end{array}\right)\left(\begin{array}{c}
N-F \\
M-i
\end{array}\right)}{\left(\begin{array}{c}
N \\
M
\end{array}\right)}
$$

Since this value is usually very close to zero, we have used $-\log _{10}(P$-value $)$ to denote the enrichment. For the later analysis of overlap between the set of genes derived as homologs from other eukaryotic species namely $S p$ and $S c$, the $N$ variable is limited to the number of genes in that species that have clear orthologs in Fg. Similarly, $\boldsymbol{F}$ and $\boldsymbol{M}$ are also reduced to only include genes with clear orthologs in Fg. Since only about a quarter of genes have orthologs in $F g$, this reduction allows us to compute the effective enrichment without biasing the function towards the genes that are present in set but have no orthologs in $\mathrm{Fg}$.

\section{Determining one-to-one orthologous genes}

We derived orthologous genes from $S c$ and $S p$ to the genes in $\mathrm{Fg}$ for our gene set enrichment analysis based on a oneto-one map from yeast genes to the gene in $\mathrm{Fg}$ according to the highest similarity score of Blastp $\left(\mathrm{E} \leq 10^{-5}\right)$.

\section{Strains and materials}

The sequenced strain of F. graminearum (PH-1; [5]) was used for expression analysis. Gene expression levels were determined using a custom Fusarium graminearum Affymetrix microarray (Fusariuma520094; [11]). Data from a spore germination time-course were used to create expression clusters [10] and raw data are available as experiment FG7 at the database PLEXdb [43]. K-means clustering was performed in the Analyst module of Expressionist version 5.1 (Genedata) using raw data imported and normalized according to [10]. The silhouette score was used to determine the optimum number of clusters for genes in each GO category using a specified range of 2-5. Default settings were used for distance, centroid calculation and maximum iterations fields.

Additional file 1: Supplementary methods on motif discovery. This file contains a more detailed description of the methods used in motif discovery.

\section{Acknowledgements}

We thank the Broad Institute for making the genomes of the three Fusarium species available before publication. LK and LJM are supported by USDA 2005-35600-16405; CK and AB are supported by NSF EnGen 0723451; and $\mathrm{XX}$ is supported by NSF DBI0846218. 


\section{Author details}

${ }^{1}$ Broad Institute, Cambridge, MA, USA. ${ }^{2}$ USDA ARS Cereal Disease Laboratory, University of Minnesota, St Paul, MN, USA. ${ }^{3}$ Department of Computer Science, Institute for Genomics and Bioinformatics, University of California, Irvine, CA, USA.

\section{Authors' contributions}

LK implemented and executed the computational tools for motif discovery and promoter enrichment analyses; $A B$ performed the gene expression and clustering analysis; CK, LM and XX designed and coordinated the project; all authors wrote the paper; all authors read and approved the final manuscript.

Received: 9 September 2009 Accepted: 26 March 2010

Published: 26 March 2010

\section{References}

1. O'Donnell K, Sutton DA, Rinaldi MG, Magnon KC, Cox PA, Revankar SG, Sanche S, Geiser DM, Juba JH, van Burik JAH, et al: Genetic diversity of human pathogenic members of the Fusarium oxysporum complex inferred from multilocus DNA sequence data and amplified fragment length polymorphism analyses: Evidence for the recent dispersion of a geographically widespread clonal lineage and nosocomial origin. Journal of Clinical Microbiology 2004, 42(11):5109-5120.

2. Bai GH, Shaner G: Management and resistance in wheat and barley to Fusarium head blight. Annual Review of Phytopathology 2004, 42:135-161

3. Frandsen RJ, Nielsen NJ, Maolanon N, Sorensen JC, Olsson S, Nielsen J, Giese H: The biosynthetic pathway for aurofusarin in Fusarium graminearum reveals a close link between the naphthoquinones and naphthopyrones. Mol Microbiol 2006, 61(4):1069-1080.

4. Coleman JJ, Rodriguez-Carres S, Kuo A, Wasmann CC, Grimwood J, J. Schmutz M, Taga M, White GJ, Zhou S, Schwartz DC, et al: The genome of Nectria haematococca: contribution of supernumerary chromosomes to gene expansion. PLoS Genet 2009, 5(8):e1000618.

5. Cuomo CA, Guldener U, Xu JR, Trail F, Turgeon BG, Di Pietro A, Walton JD, Ma $L$, Baker SE, Rep M, et al: The Fusarium graminearum genome reveals a link between localized polymorphism and pathogen specialization. science 2007, 317(5843):1400-1402.

6. Guldener U, Mannhaupt G, Munsterkotter M, Haase D, Oesterheld M, Stumpflen V, Mewes HW, Adam G: FGDB: a comprehensive fungal genome resource on the plant pathogen Fusarium graminearum. Nucleic Acids Res 2006, 34 Database: D456-458.

7. Guldener U, Seong KY, Boddu J, Cho S, Trail F, Xu JR, Adam G, Mewes HW, Muehlbauer GJ, Kistler HC: Development of a Fusarium graminearum Affymetrix GeneChip for profiling fungal gene expression in vitro and in planta. Fungal Genet Biol 2006, 43(5):316-325.

8. Ma $\sqcup$, Does van der HC, Borkovich KA, Coleman JJ, Daboussi MJ, D Pietro A, Dufresne M, Freitag M, Grabherr M, Henrissat B, et al: Comparative genomics reveals mobile pathogenicity chromosomes in Fusarium. Nature 2010, 464:367-373.

9. Seong KY, Pasquali M, Zhou XY, Song J, Hilburn K, McCormick S, Dong YH, Xu JR, Kistler HC: Global gene regulation by Fusarium transcription factors Tri6 and Tri10 reveals adaptations for toxin biosynthesis. Molecular Microbiology 2009, 72(2):354-367.

10. Seong KY, Zhao X, Xu JR, Guldener U, Kistler HC: Conidial germination in the filamentous fungus Fusarium graminearum. Fungal Genetics and Biology 2008, 45(4):389-399.

11. Guldener U, Mannhaupt G, Munsterkotter M, Haase D, Oesterheld M, Stumpflen V, Mewes HW, Adam G: FGDB: a comprehensive fungal genome resource on the plant pathogen Fusarium graminearum. Nucleic Acids Research 2006, 34:D456-D458.

12. Kellis M, Patterson N, Endrizzi M, Birren B, Lander ES: Sequencing and comparison of yeast species to identify genes and regulatory elements. Nature 2003, 423(6937):241-254

13. Wu J, Sieglaff DH, Gervin J, Xie XS: Discovering regulatory motifs in the Plasmodium genome using comparative genomics. Bioinformatics 2008, 24(17):1843-1849.

14. Stark A, Lin MF, Kheradpour P, Pedersen JS, Parts L, Carlson JW, Crosby MA, Rasmussen MD, Roy $S$, Deoras AN, et al: Discovery of functional elements in 12 Drosophila genomes using evolutionary signatures. Nature 2007, 450(7167):219-232.
15. Sieglaff DH, Dunn WA, Xie XHS, Megy K, Marinotti O, James AA: Comparative genomics allows the discovery of cis-regulatory elements in mosquitoes. Proceedings of the National Academy of Sciences of the United States of America 2009, 106(9):3053-3058.

16. Elemento O, Tavazoie S: Fast and systematic genome-wide discovery of conserved regulatory elements using a non-alignment based approach. Genome Biology 2005, 6(2):R18.

17. Ettwiller L, Paten B, Souren M, Loosli F, Wittbrodt J, Birney E: The discovery, positioning and verification of a set of transcription-associated motifs in vertebrates. Genome Biol 2005, 6(12):R104.

18. Xie XH, Lu J, Kulbokas EJ, Golub TR, Mootha V, Lindblad-Toh K, Lander ES, Kellis M: Systematic discovery of regulatory motifs in human promoters and 3 ' UTRs by comparison of several mammals. Nature 2005, 434(7031):338-345.

19. Stormo GD: DNA binding sites: representation and discovery. Bioinformatics 2000, 16(1):16-23.

20. Ashburner M, Ball CA, Blake JA, Botstein D, Butler H, Cherry JM, Davis AP, Dolinski K, Dwight SS, Eppig JT, Harris MA, Hill DP, Issel-Tarver L, Kasarskis A, Lewis S, Matese JC, Richardson JE, Ringwald M, Rubin GM, Sherlock G: Gene ontology: tool for the unification of biology. The Gene Ontology Consortium. Nat Genet 2000, 25(1):25-29.

21. Fischer R: The cytoskeleton and polarized growth of filamentous fungi. Biology of the Fungal Cell New York: SpringerHoward RJ, Gow NAR 2007, 121-135

22. Mahony S, Benos PV: STAMP: a web tool for exploring DNA-binding motif similarities. Nucleic Acids Res 2007, 35 Web Server: W253-258.

23. Maclsaac KD, Wang T, Gordon DB, Gifford DK, Stormo GD, Fraenkel E: An improved map of conserved regulatory sites for Saccharomyces cerevisiae. BMC Bioinformatics 2006, 7:113.

24. Ogawa N, DeRisi J, Brown PO: New components of a system for phosphate accumulation and polyphosphate metabolism in Saccharomyces cerevisiae revealed by genomic expression analysis. $\mathrm{Mol}$ Biol Cell 2000, 11(12):4309-4321.

25. Oshima Y: The phosphatase system in Saccharomyces cerevisiae. Genes Genet Syst 1997, 72(6):323-334.

26. Brasaemle DL: Thematic review series: adipocyte biology. The perilipin family of structural lipid droplet proteins: stabilization of lipid droplets and control of lipolysis. Journal of Lipid Research 2007, 48(12):2547-2559.

27. Caddick MX, Brownlee AG, Arst HN Jr: Phosphatase regulation in Aspergillus nidulans: responses to nutritional starvation. Genet Res 1986, 47(2):93-102

28. Kang S, Metzenberg RL: Molecular analysis of nuc-1+, a gene controlling phosphorus acquisition in Neurospora crassa. Mol Cell Biol 1990, 10(11):5839-5848.

29. Leal J Squina FM, Freitas JS, Silva EM, Ono CJ, Martinez-Rossi NM, Rossi A: A splice variant of the Neurospora crassa hex-1 transcript, which encodes the major protein of the Woronin body, is modulated by extracellular phosphate and pH changes. FEBS Lett 2009, 583(1):180-184.

30. Proft M, Pascual-Ahuir A, de Nadal E, Arino J, Serrano R, Posas F: Regulation of the Sko1 transcriptional repressor by the Hog1 MAP kinase in response to osmotic stress. EMBO J 2001, 20(5):1123-1133

31. Gasch AP, Spellman PT, Kao CM, Carmel-Harel O, Eisen MB, Storz G, Botstein D, Brown PO: Genomic expression programs in the response of yeast cells to environmental changes. Mol Biol Cell 2000, 11(12):4241-4257.

32. Chen D, Toone WM, Mata J, Lyne R, Burns G, Kivinen K, Brazma A, Jones N, Bahler J: Global transcriptional responses of fission yeast to environmental stress. Mol Biol Cell 2003, 14(1):214-229.

33. Nikolaou E, Agrafioti I, Stumpf M, Quinn J, Stansfield I, Brown AJP: Phylogenetic diversity of stress signalling pathways in fungi. Bmc Evolutionary Biology 2009, 9:44-45

34. Warner JR: The economics of ribosome biosynthesis in yeast. Trends in Biochemical Sciences 1999, 24(11):437-440.

35. Panozzo C, Cornillot E, Felenbok BA: The CreA repressor is the sole DNAbinding protein responsible for carbon catabolite repression of the alc $A$ gene in Aspergillus nidulans via its binding to a couple of specific sites. Journal of Biological Chemistry 1998, 273(11):6367-6372.

36. Scazzocchio C: The fungal GATA factors. Curr Opin Microbiol 2000, 3(2):126-31.

37. Guo Z, Sherman F: $3^{\prime}$-end-forming signals of yeast mRNA. Mol Cell Biol 1995, 15(11):5983-5990. 
38. Irniger S, Egli CM, Braus GH: Messenger RNA 3'-end formation of a DNA fragment from the human c-myc 3'-end region in Saccharomyces cerevisiae. Curr Genet 1993, 23(3):201-204.

39. Tanay A, Regev A, Shamir R: Conservation and evolvability in regulatory networks: the evolution of ribosomal regulation in yeast. Proc Natl Acad Sci USA 2005, 102(20):7203-8.

40. Gasch AP, Moses AM, Chiang DY, Fraser HB, Berardini M, Eisen MB: Conservation and evolution of cis-regulatory systems in ascomycete fungi. PLoS Biol 2004, 2(12):e398.

41. Li M, Ma B, Kisman D, Tromp J: Patternhunter II: highly sensitive and fast homology search. J Bioinform Comput Biol 2004, 2(3):417-439.

42. Brudno M, Do CB, Cooper GM, Kim MF, Davydov E, Green ED, Sidow A, Batzoglou S: LAGAN and Multi-LAGAN: efficient tools for large-scale multiple alignment of genomic DNA. Genome Res 2003, 13(4):721-731.

43. Wise RP, Caldo RA, Hong L, Shen L, Cannon E, Dickerson JA: BarleyBase/ PLEXdb. Methods Mol Biol 2007, 406:347-363.

doi:10.1186/1471-2164-11-208

Cite this article as: Kumar et al:: Systematic discovery of regulatory

motifs in Fusarium graminearum by comparing four Fusarium genomes. BMC Genomics 2010 11:208.

\section{Submit your next manuscript to BioMed Central} and take full advantage of:

- Convenient online submission

- Thorough peer review

- No space constraints or color figure charges

- Immediate publication on acceptance

- Inclusion in PubMed, CAS, Scopus and Google Scholar

- Research which is freely available for redistribution

Submit your manuscript at www.biomedcentral.com/submit 